\title{
Predicting Body Composition in College Students Using the Womersley and Durnin Body Mass Index Equation
}

Jeremy P. Loenneke ${ }^{* 1 B C D G}$, MS; Kathryn M. Hirt ${ }^{\text {2CDG }}$, MS; Jacob M. Wilson ${ }^{3 \mathrm{CDG}}$, PhD; Jeremy T Barnes ${ }^{4 A B D G}$, PhD; Thomas J Pujol ${ }^{4 A B D G}$, EdD

\author{
Authors' Affiliation: \\ 1. Department of Health and Exercise \\ Science. The University of Oklahoma, \\ Norman, Oklahoma, USA \\ 2. Cooper Aerobics, Oklahoma City, \\ Oklahoma, USA \\ 3. Department of Health Sciences and \\ Human Performance, University of \\ Tampa, Tampa, Florida, USA \\ 4. Department of Health, Human \\ Performance, and Recreation. Southeast \\ Missouri State University, Cape \\ Girardeau, Missouri, USA \\ Authors' Contribution \\ A Concept / Design \\ B Acquisition of Data \\ C Data Analysis / Interpretation \\ D Manuscript Preparation \\ E Critical Revision of the Manuscrip \\ F Funds Collection \\ G Approval of the Article

\section{* Corresponding Author; Address: 1401 Asp Avenue, 104 Huston Huffman Center. Norman, Oklahoma 73019-0615, USA} \\ E-mail: jploenneke@ou.edu \\ Received: Aug 16, 2012 \\ Accepted: Feb 12, 2013 \\ Available Online: Feb 16, 2013
}

\begin{abstract}
Purpose: When assessing fitness levels, body composition is usually measured. The purpose of this study was to determine the overall efficacy of a body mass index (BMI) equation for predicting body composition with respect to college aged participants.
\end{abstract}

Methods: Body composition was measured using dual-energy x-ray absorptiometry (DXA) and was estimated using the Womersley and Durnin BMI prediction equation.

Results: There was no significant $(P=0.8)$ percent body fat $(\% \mathrm{BF})$ difference between the BMI prediction equation and DXA (BMI Predicted=25 (10) $[\min =6 ; \max =52] \% B F$ vs DXA=25 (6) $[\min =10 ; \max =45]$ \%BF). In addition, a significant correlation was found between the two approaches $(r=0.791$, $P=0.001)$. However, both the standard error of estimate $(6.32 \% \mathrm{BF})$ and total error $(6.63 \% \mathrm{BF})$ were outside acceptable ranges for prediction equations.

Conclusion: The Womersley and Durnin equation for estimating \%BF was not found to be a good estimate. Therefore, although the BMI predicted \%BF has been previously found to predict skinfold estimated \%BF, it does not appear valid in estimating \%BF from DXA.

Key Words: Body Fat; Percent Fat; Fat Mass

\section{INTRODUCTION}

$\mathrm{W}$ hen assessing fitness levels, body composition is usually measured. Numerous methods such as skinfolds, bioelectrical impedance analysis (BIA), underwater weighing and dual-energy $\mathrm{x}$-ray absorptiometry (DXA) have been used to estimate body composition ${ }^{[1-3]}$. All have advantages and disadvantages in terms of predictability and accuracy ${ }^{[4]}$. Cross-sectional imaging techniques such as magnetic resonance imaging (MRI) and computed tomography (CT) are currently the most precise measures available, allowing differentiation of subcutaneous adipose tissue (SAT) and visceral adipose tissue (VAT), and can measure changes in these compartments ${ }^{[5,6]}$. Despite the precision, CT relies on $\mathrm{x}$-ray radiation for imaging at levels higher than that seen with DXA, which limits its widespread repeated use. While MRI does not use radiation, it is limited by both cost and time ${ }^{[7]}$. All of these aforementioned methods, while highly precise, are inconvenient to most practitioners, particularly those who are looking for a quick, convenient, method to estimate body composition.

Some research has focused on field methods of estimating body composition particularly arm-to-arm or leg-to-leg ${ }^{[8,9]}$ BIA and skin folds ${ }^{[10-12]}$. Both methods are significantly influenced by outside factors, which are hard to control for when screening a large number of participants. For instance, for an accurate assessment of body fat percentage $(\% \mathrm{BF})$ utilizing $\mathrm{BIA}$, it is advisable to have the person fast overnight, go 24 
hours without exercise or alcohol while maintaining normal levels of hydration ${ }^{[13]}$, which are often overlooked by both the researcher and practitioner. Skinfolds have shown to be as precise as the DXA in estimating $\% \mathrm{BF}$, however this too assumes that the technician is trained with locating and measuring skin fold thickness at the correct anatomical points ${ }^{[13]}$. Maintaining both inter- and intra-technician reliability is also a concern.

Body mass index (BMI) has been adopted widely as a measure of obesity ${ }^{[14]}$, yet its extrapolation for the general population is not without limitations. In large scale studies where subjects are asked to recall their height and weight, subjects often overestimate height and underestimate their weight, resulting in a lower BMI ${ }^{[15,16]}$. BMI is also limited by the inability to discriminate between fat and lean mass. In spite of this, validation studies show high correlations between BMI and $\% \mathrm{BF}^{[17]}$. To provide a better estimate, Womersley and Durnin ${ }^{[18]}$ developed a prediction equation which used BMI and has been previously observed to accurately predict skinfold estimated $\% \mathrm{BF}$ across ages 17-72. The overall efficacy of this equation with respect to DXA estimated \% $\mathrm{BF}$ in college students is currently unknown. This equation from 1977 is being reinvestigated to determine if this could offer a costeffective technique of predicting $\% \mathrm{BF}$, independent of the practitioner's skill level.

\section{METHODS AND SUBJECTS}

One hundred and twenty nine college students (male: $\mathrm{n}=63$; female: $\mathrm{n}=66$ ) volunteered to participate in this study. Subjects attended the laboratory on one occasion and were thoroughly informed of the purpose, nature, practical details and possible risks associated with the experiment, as well as the right to terminate participation at will, before they gave their voluntary informed consent to participate. The study was approved by the University's institutional review board.

The subject's criterion body composition was estimated using a GE Lunar Prodigy DXA machine
(GE Healthcare, Pewaukee, WI). Each day before testing, a quality assurance phantom was performed and passed. Before each test, the subjects' height was measured to the nearest $\mathrm{cm}$ using a wall-mounted stadiometer and body mass was measured using an electronic scale (Tanita BF-350, Arlington Heights, Illinois). These variables together with sex and ethnicity were entered into the DXA software. Subjects lied supine on the DXA table with their hands lying flat and pronated. Prior to the DXA scan subjects were asked to refrain from eating for 2-3 hours and were asked to void immediately prior to their test. Females were required to complete an over the counter early pregnancy test prior to participation. Lunar software algorithms calculated estimates of $\% \mathrm{BF}$ for each subject.

\section{BMI Prediction Equation:}

Subjects' \%BF was estimated using a prediction equation developed by Womersley and Durnin ${ }^{[1]}$. Below are listed the equations for males and females respectively. BMI is the only unknown variable, whereas all others are known constants.

Male $\% \mathrm{BF}=1.34 * \mathrm{BMI}-12.47$

Female $\% \mathrm{BF}=1.37 * \mathrm{BMI}-3.47$.

\section{Statistical analyses:}

Male and female data were pooled together, because the equations themselves account for gender differences. The validity of the $\% \mathrm{BF}$ estimates was based on the evaluation of the BMI prediction equation versus the estimated value from the DXA by calculating the mean, SD, Pearson correlation, and standard error of estimate (SEE) from linear regression analysis. SEE represents the degree of deviation of individual scores from the regression line. To assess the average deviation of individual scores from the line of identity, total error (TE) was calculated for each field method. Paired t-tests determined pair-wise differences between measurements using an alpha level of 0.05. Differences between each method were plotted in Excel (Microsoft, Redmond, WA, USA) against their group mean ((BMI Prediction-DXA mean)/2) to determine the directional bias of the BMI estimate. All other statistical analyses were made using PASW Statistics 18, with all variability represented using standard deviation (SD). 
Table 1: Descriptive data of study participants $(n=129)$

\begin{tabular}{lccc} 
Parameter & Mean (SD) & Minimum & Maximum \\
\hline Age (yr.) & $21(2)$ & 18 & 33 \\
Height (cm) & $172.1(10.9)$ & 142.2 & 203.2 \\
Body mass (kg) & $74.5(15.9)$ & 47.7 & 123.6 \\
Body Mass Index (kg/m $\mathbf{2})$ & $24.9(3.7)$ & 16.9 & 35.8
\end{tabular}

\section{RESULTS}

Subject characteristics are found in Table 1. There were no significant $(p=0.783)$ mean differences for $\% \mathrm{BF}$ between the BMI prediction equation and the DXA estimate (Figure 1). In addition, there was a significant $(\mathrm{p}<0.001)$ high correlation between the two estimates for $\% \mathrm{BF}$ (Figure 2). Although there were no mean differences between estimates, the average deviation of the individual scores from the regression line (SEE) was $6.2 \% \mathrm{BF}$ and the average deviation of the individual scores from the line of identity (TE) was $6.6 \% \mathrm{BF}$. Both the SEE and TE were outside the acceptable ranges for prediction equations according to Lohman ${ }^{[18]}$. Moreover, the BMI prediction equation for $\% \mathrm{BF}$ overestimated the lean and underestimated those who were less lean (Fig. 3).

Using the current data, we predicted the DXA estimate for $\% \mathrm{BF}$ using the predictors $\mathrm{BMI}$ and sex. The following equation had a SEE of $6.2 \% \mathrm{BF}$ and the proportion of variance explained by the set of predictors was $64 \%$.

$$
\begin{aligned}
& y=1.924(x)+13.223(z)-28.966 . \\
& y=\% B F \\
& x=B M I \\
& z=\operatorname{Sex}(0=\text { Male, } 1=\text { Female })
\end{aligned}
$$

\section{DISCUSSION}

BMI calculated using a person's height and weight does not require a high level of expertise for measurements and the equation, if valid, would allow for an affordable, quick, and accurate estimate of body composition for college students. However, although the prediction equation provided a group mean value which was not different than the body composition estimated by the DXA; the SEE and TE were outside

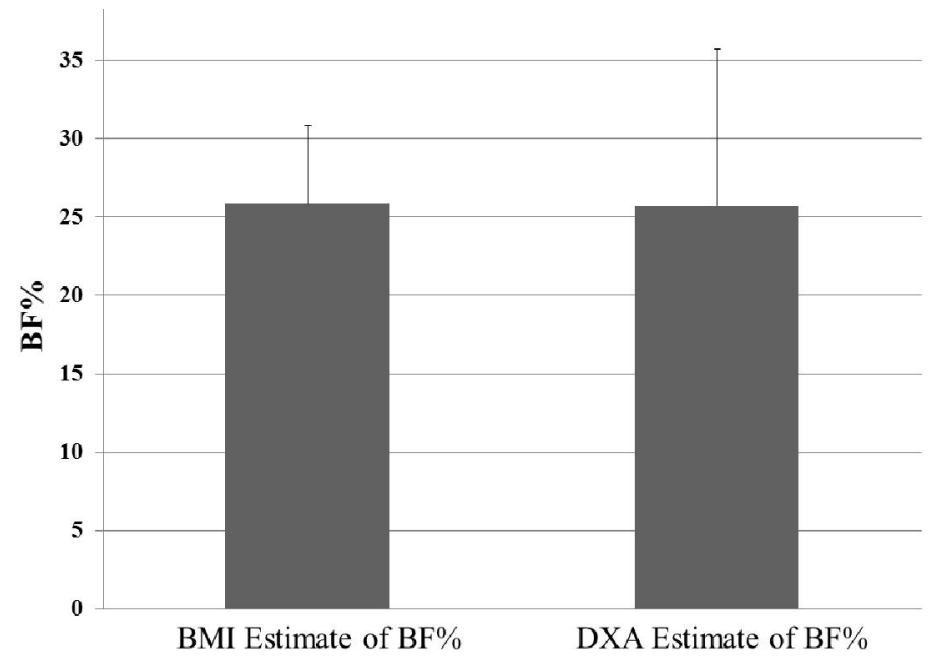

Fig. 1: Differences in percent body fat $(\% \mathrm{BF})$ between the body mass index (BMI) prediction equation and the dual energy X-ray absorptiometry (DXA). Estimates are presented as means (Standard Deviation). 


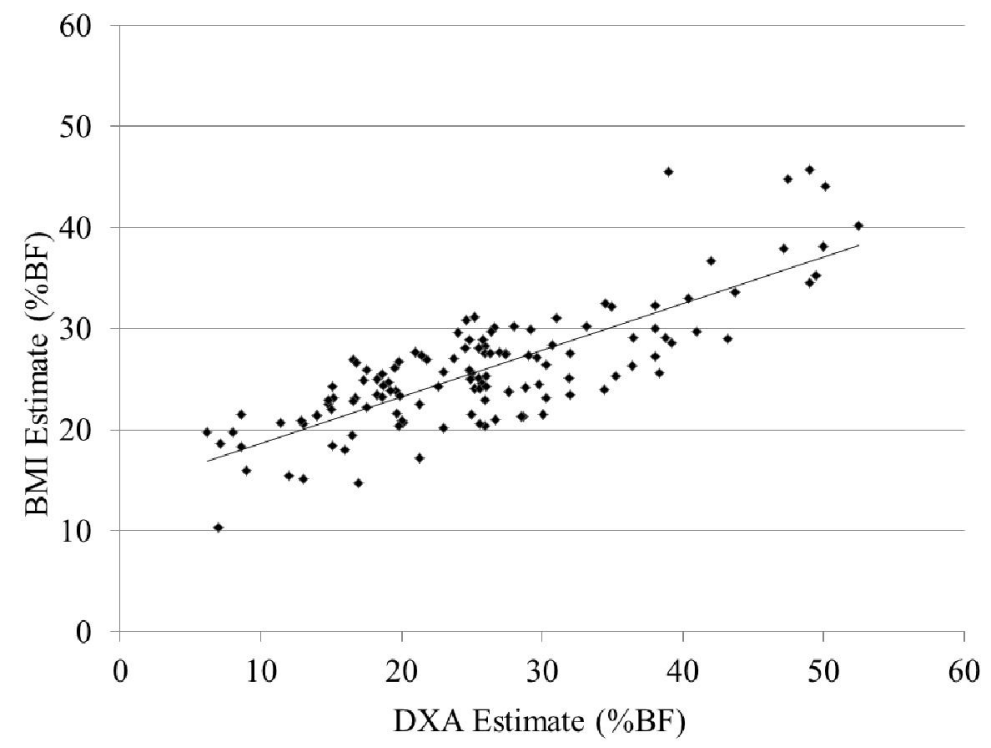

Fig. 2: The relationship between the body mass index (BMI) prediction equation and the dual energy X-ray absorptiometry (DXA) for percent body fat (\%BF).

the acceptable ranges according to Lohman ${ }^{[19]}$. In addition, the BMI equation tended to overestimate $\% \mathrm{BF}$ in those who were leaner and underestimate \%BF in those who were less lean according to the DXA estimate Therefore, in this cohort of collegiate males and females, the formula does not appear to provide an accurate estimate of \%BF. Therefore, although BMI may provide a reasonable estimate of disease risk, it may not necessarily reflect the level of $\% \mathrm{BF}$ in college aged young adults. In addition, our prediction equation for DXA \%BF using the predictors BMI and sex provided a similar SEE as the BMI estimate from Womersley and Durnin equation. Therefore, neither equation produces acceptable deviations from the

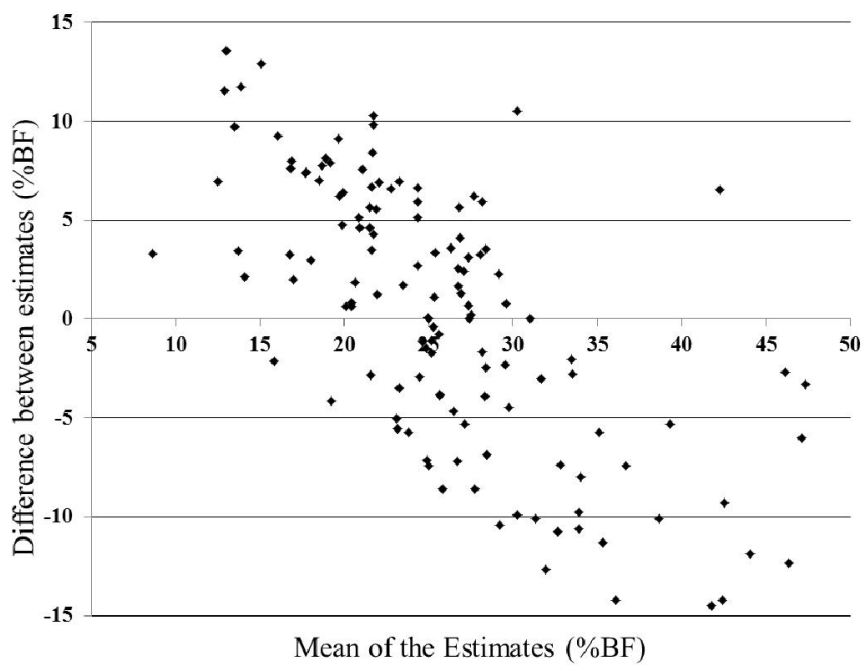

Fig. 3: Differences in percent body fat $(\% \mathrm{BF})$ between the body mass index (BMI) prediction equation and the dual energy X-ray absorptiometry (DXA) were plotted against their group mean ((BMI prediction mean+DXA mean)/2) to determine the directional bias of the BMI estimate compared to the DXA. 
regression line ( $\leq 4 \mathrm{SEE})$.

Noted limitations of this study include the use of DXA as our criterion method for estimating \%BF rather than the gold standard estimate of hydrostatic weighing. In addition, although we encouraged the participants to arrive at the laboratory in a hydrated state, no quantitative measure of hydration was taken. This is an important limitation to note as the DXA assumes a certain percentage of body water. In addition, we used DXA and did not measure skinfold thickness, therefore we are unable to determine directly if the Womersley and Durnin ${ }^{[18]}$ equation is valid in collegiate males and females. Instead, we can only state that the BMI prediction equation developed to predict skinfolds, does not appear valid in predicting $\% \mathrm{BF}$ estimated from the DXA.

\section{CONCLUSION}

The Womersley and Durnin ${ }^{[18]}$ equation for estimating body composition was not found to be a good estimate. Therefore, although the BMI predicted \%BF has been previously found to predict skinfold estimated $\% \mathrm{BF}$, it does not appear valid in estimating \%BF from DXA. The results of this study do not support the use of the Womersley and Durnin ${ }^{[18]}$ equation.

\section{ACKNOWLEDGMENTS}

This manuscript was not supported by any funding. Data collected from the Human Performance Lab at Southeast Missouri State University

Conflict of interests: None

\section{REFERENCES}

[1] Mazess RB, Barden HS, Bisek JP, et al. Dual-energy x-ray absorptiometry for total-body and regional bone-mineral and soft-tissue composition. Am J Clin Nutr 1990;51:1106-12.

[2] Wang J, Heymsfield SB, Aulet M, et al. Body fat from body density: underwater weighing vs. dual-photon absorptiometry. Am J Physiol 1989;256:E829-34.

[3] Loenneke JP, Wilson JM, Wray ME, et al. The estimation of the fat free mass index in athletes. Asian J Sports Med 2012;3:200-3.

[4] Norgan NG. Laboratory and field measurements of body composition. Public Health Nutr 2005;8:1108-22.

[5] Mayo-Smith W, Hayes CW, Biller BM, et al. Body fat distribution measured with CT: correlations in healthy subjects, patients with anorexia nervosa, and patients with Cushing syndrome. Radiology 1989;170:515-8.

[6] Ross R, Leger L, Morris D, et al. Quantification of adipose tissue by MRI: relationship with anthropometric variables. J Appl Physiol 1992;72:787-95.

[7] Wagner DR, Heyward VH. Techniques of body composition assessment: a review of laboratory and field methods. Res $Q$ Exerc Sport 1999;70:135-49.

[8] Pateyjohns IR, Brinkworth GD, Buckley JD, et al. Comparison of three bioelectrical impedance methods with DXA in overweight and obese men. Obesity (Silver Spring) 2006;14:2064-70.

[9] Cable A, Nieman DC, Austin M, et al. Validity of leg-to-leg bioelectrical impedance measurement in males. J Sports Med Phys Fitness 2001;41:411-4.

[10] Ball S, Swan PD, DeSimone R. Comparison of anthropometry to dual energy X-ray absorptiometry: a new prediction equation for women. Res Q Exerc Sport 2004;75:248-58.

[11] Jackson AS, Pollock ML. Generalized equations for predicting body density of men. Br J Nutr 1978;40:497-504.

[12] Jackson AS, Pollock ML, Ward A. Generalized equations for predicting body density of women. Med Sci Sports Exerc 1980;12:175-81.

[13] Fuller NJ, Jebb SA, Laskey MA, et al. Four-component model for the assessment of body composition in humans: comparison with alternative methods, and evaluation of the density and hydration of fat-free mass. Clin Sci (Lond) 1992;82:687-93.

[14] WHO. Physical status: the use and interpretation of anthropometry. Report of a WHO Expert Committee. World Health Organ Tech Rep Ser 1995;854:1-452.

[15] Moore SC, Patel AV, Matthews CE, et al. Leisure time physical activity of moderate to vigorous intensity and mortality: a large pooled cohort analysis. PLoS Med 2012;9:e1001335.

[16] Shields M, Gorber SC, Tremblay MS. Effects of measurement on obesity and morbidity. Health Rep 2008;19:77-84.

[17] Sun Q, van Dam RM, Spiegelman D, et al. Comparison of dual-energy x-ray absorptiometric and anthropometric measures of adiposity in relation to adiposity-related biologic factors. Am J Epidemiol 2010;172:1442-54.

[18] Womersley J, Durnin J. A comparison of the skinfold method with extent of 'overweight' and various weight-height relationships in the assessment of obesity. Br J Nutr 1977;38:271-84.

[19] Lohman TG. Advances in body composition assessment. Champaign, IL: Human Kinetics; 1992. 